\title{
NEUROIMAGING
}

\section{Utility of Double Inversion Recovery Sequences in MRI}

\author{
Maura E. Ryan, $\mathrm{MD}^{1,2 *}$ \\ ${ }^{I}$ Division of Neuroradiology, Department of Medical Imaging, Ann \& Robert H. Lurie Children's Hospital of Chicago, Chicago, IL \\ ${ }^{2}$ Department of Radiology, Northwestern University Feinberg School of Medicine, Chicago, IL \\ *Correspondence: Dr. Maura E. Ryan, E-mail: mryan@luriechildrens.org
}

Related Article: Wong-Kisiel LC, Britton JW, Witte RJ, Kelly-Williams KM, Kotsenas AL, Krecke KN et al. Double Inversion Recovery Magnetic Resonance Imaging in Identifying Focal Cortical Dysplasia. Pediatr Neurol 2016 Aug;61:87-93.

Keywords: Double Inversion Recovery MRI; Focal cortical dysplasia; Epilepsy

Investigators from the Mayo Clinic, Rochester Minnesota investigated the utility of three-dimensional (3D) double inversion recovery (DIR) sequences in magnetic resonance imaging (MRI) detection of focal cortical dysplasia (FCD) in children and young adults with epilepsy. Sixteen patients with FCD, 13 patients with periventricular heterotopia and 20 normal patients were evaluated with DIR sequences as well as standard epilepsy MRI 3D magnetization prepared rapid acquired gradient echoes (MPRAGE) sequences. All but one study was performed on a 3 Tesla (T) magnet.

DIR and MPRAGE sequences were independently reviewed by board certified neuroradiologists blinded to the clinical patient data, and scored as normal, indicative of FCD, or periventricular heterotopia. The sensitivity and specificity of the DIR sequence for FCD among all observers ranged from $50-88 \%$ and $67-91 \%$ respectively, although the consensus among readers with greater experience yielded an $88 \%$ sensitivity and $88 \%$ specificity for the DIR sequence, compared with a $44 \%$ sensitivity and $100 \%$ specificity of for the MPRAGE sequence. The authors concluded that the DIR sequence is sensitive for the detection of focal cortical dysplasia, particularly when reviewed by experienced interpreters, and may be a useful adjunct in MRI imaging of suspected structural epilepsy. [1]

COMMENTARY. FCDs are a possible cause of lesional epilepsy and are potentially curable with resection. Although identification of FCDs by imaging is associated with better post-surgical outcomes, some types of FCDs may be exceedingly subtle or undetectable by MRI, even with dedicated high resolution sequences on high field MRI [2].

Inversion recovery is a MRI technique used to suppress signal from specific tissue or fluid types. DIR sequences suppress both cerebrospinal fluid (CSF) signal and normal white matter signal. This may make abnormal white matter associated with FCDs, such as signal changes at the gray-white junction and radial bands extending toward the ventricular margin, more apparent.

Other studies have also shown that some FCD lesions may be better seen with DIR sequences than with standard MRI sequences such as T2-weighted fluid attenuation inversion recovery (FLAIR) images, which suppress only CSF signal, and 3D MPRAGE [3,4]. DIR sequences have also been shown to improve detection of other parenchymal changes such as demyelinating plaques of multiple sclerosis [5].

However, it should be noted that DIR sequences also require a relatively longer acquisition time, are prone to CSF pulsation and vascular flow artifact, and interpretation is partially dependent on the experience of the reader. Artifacts likely account for the observed lower specificity of DIR sequences for FCD. Additionally, this sequence has a relatively low signal to noise ratio, which likely reduces the sensitivity at lower, standard 1.5T, magnet strength [5]. Still, preliminary data suggests DIR imaging may be a useful compliment to traditional 3T MRI sequences in patients with epilepsy where subtle FCDs are clinically suspected.

\section{Disclosures}

The author(s) have declared that no competing interests exist.

\section{References}

1. Wong-Kisiel LC, Britton JW, Witte RJ, Kelly-Williams KM, Kotsenas $\mathrm{AL}, \mathrm{Krecke} \mathrm{KN}$ et al. Double Inversion Recovery Magnetic Resonance Imaging in Identifying Focal Cortical Dysplasia. Pediatr Neurol 2016 Aug;61:87-93. http://dx.doi.org/10.1016/j.pediatrneurol.2016.04.013 PMID:27241231

2. Wang ZI, Alexopoulos AV, Jones SE, Jaisani Z, Najm IM, Prayson RA. The pathology of magnetic-resonance-imaging-negative epilepsy. Mod Pathol 2013 Aug;26(8):1051-8. http://dx.doi.org/10.1038/modpathol .2013.52 PMID:23558575

3. Granata F, Morabito R, Mormina E, Alafaci C, Marino S, Laganà A et al. 3T Double Inversion Recovery Magnetic Resonance Imaging: diagnostic advantages in the evaluation of cortical development anomalies. Eur J Radiol 2016 May;85(5):906-14. http://dx.doi.org/10.1016/j.ejrad.2016. 02.018 PMID:27130050

4. Soares BP, Porter SG, Saindane AM, Dehkharghani S, Desai NK. Utility of double inversion recovery MRI in paediatric epilepsy. Br J Radiol 2016;89(1057):20150325. http://dx.doi.org/10.1259/bjr.20150325 PMID:26529229

5. Wattjes MP, Lutterbey GG, Gieseke J, Träber F, Klotz L, Schmidt S et al. Double inversion recovery brain imaging at 3T: diagnostic value in the detection of multiple sclerosis lesions. AJNR Am J Neuroradiol 2007 Jan;28(1):54-9. PMID:17213424 